Research Article

\title{
Error Distribution of a 5-Axis Measuring Machine Based on Sensitivity Analysis of Geometric Errors
}

\author{
Xicong Zou $\mathbb{D}^{\circ},{ }^{1}$ Xuesen Zhao $\mathbb{C}^{\circ},{ }^{2}$ Zongwei Wang, ${ }^{3}$ Guo $\mathrm{Li}^{3}$ Zhenjiang $\mathrm{Hu},{ }^{2}$ and Tao Sun ${ }^{2}{ }^{2}$ \\ ${ }^{1}$ School of Mechatronics Engineering, Heilongjiang University, Harbin 150080, China \\ ${ }^{2}$ Center for Precision Engineering, Harbin Institute of Technology, Harbin 150001, China \\ ${ }^{3}$ Research Center of Laser Fusion, China Academy of Engineering Physics, Mianyang 621900, China
}

Correspondence should be addressed to Xuesen Zhao; zhaoxuesen@hit.edu.cn and Tao Sun; taosun@hit.edu.cn

Received 8 May 2019; Revised 30 August 2019; Accepted 23 October 2019; Published 14 February 2020

Academic Editor: Francesco Aggogeri

Copyright ( $\odot 2020$ Xicong Zou et al. This is an open access article distributed under the Creative Commons Attribution License, which permits unrestricted use, distribution, and reproduction in any medium, provided the original work is properly cited.

Geometric errors are inevitably introduced into any multiaxis measuring system, and the geometric error is one of the main factors that seriously affects the measurement accuracy. The present work investigates the error distribution of the prototype of a 5-axis measuring machine based on sensitivity analysis of geometric errors. The measurement error modeling of the 5axis measuring machine is first established via the homogeneous coordinate transformation, and the Sobol global sensitivity analysis method is then employed to quantify the influence of geometric errors on the measurement result with the sensitivity index. The result shows that most of the angular errors are the crucial geometric errors seriously affecting the measurement result. These errors are supposed to be fully considered in the accuracy design and manufacturing stages. The error levels of the crucial geometric errors were distributed and readjusted according to the sensitivity analysis result. Some practical approaches to distribute and improve the crucial geometric errors have been given in detail. The error distribution method is effective to equalize the influence of the crucial geometric errors on the measurement result as possible. The findings of this study provide significant meanings for the optimal design and accurate manufacturing of the 5-axis measuring machine, and the proposed method can be used to improve the measurement accuracy of the 5-axis measuring machine.

\section{Introduction}

The high machining accuracy of mechanical parts becomes increasingly essential in the fields of electronic communications, biomedicine, and aerospace [1-3]. The evaluation of machining accuracy requires the support of measurement technology, and the processing technology is supplementary to the measurement technology. The measurement technology is a comprehensive and interdisciplinary subject involving the optics, electronics, sensors, mechanical manufacturing, and computer technology; it provides evaluation and measurement methods for the processing technology. The rapid development of measurement technology is the driving force to increasingly promote the machining accuracy and vice versa.
Unfortunately, a measuring machine is not an ideal machine that operates without any error. The measuring machine inevitably operates under the influence of various error factors such as geometric errors, thermal error, and static force error, which lead to measurement inaccuracy. Geometric errors contribute serious influence to the measurement result of a measuring machine during the measuring process. Therefore, it is crucial to reveal the relationship between geometric errors and the measurement result and to further investigate the influence of geometric errors on the measurement result. The relationship between geometric errors and the measurement result can be described by the measurement error modeling based on homogeneous coordinate transformation while the influence of geometric errors on the measurement result can be obtained using the sensitivity analysis method. 
The homogeneous coordinate transformation method can accurately express the position and posture between two adjacent rigid bodies; it is very powerful to establish the measurement error modeling of a measuring machine.

Wei and Chen [4] built the geometric errors of $X$ slideway in a coordinate-measuring machine to study the effect of the geometric errors on the positioning error of the probe tip. Liu et al. [5] used homogeneous coordinate transformation to obtain the geometric error modeling of a five-axis machine tool, the geometric errors were compensated according to the geometric error modeling, and the compensation method had remarkable improvement on the machining accuracy of the machined workpiece. Yao et al. [6] obtained a geometric error modeling of an ultraprecision multiaxis machine tool and successfully found out the important error sources that contribute to the more influence on the error modeling via sensitivity analysis. Chen et al. [7] established the geometric error modeling of a five-axis ultraprecision turning machine by homogeneous coordinate transformation, and the machine tool was redesigned for optimization according to the sensitivity analysis results. Li et al. [8] described the error modeling of a five-axis machine tool based on homogeneous coordinate transformation and used a sensitivity analysis method to determine the crucial geometric error items contributing to the processing accuracy. Liu et al. $[9,10]$ investigated the geometric errors of rotary axes for a five-axis machine tool and proposed the identification and compensation method by constructing an equivalent rotary axis. A blade machining test was employed to verify the effectiveness of the proposed method.

Sensitivity analysis is one of the most effective methods to quantify the influence of each input parameter on the output result; it is classified into local sensitivity analysis and global sensitivity analysis due to the corresponding characteristic. Local sensitivity analysis can be achieved via partial differentiation and evaluates the sensitivity index of error items at a fixed position in the workspace. In contrast, global sensitivity analysis can be performed by a variety of ways including Fourier amplitude sensitivity testing (FAST) [11], Sobol method [12], and the integration method [8]. Global sensitivity analysis represents the comprehensive sensitivity over the entire workspace. Global sensitivity analysis has become the focus of attention on the research because of its excellent performance. Cheng et al. [13] employed extended Fourier amplitude sensitivity testing to investigate the interaction among geometric error items of a 3 -axis milling machine and identified the dominant geometric error items by a high-dimensional nonlinear model. Zhang et al. [14] computed the influence of geometric errors on production performance by a global sensitivity analysis method. Zhang et al. [15] utilized Sobol method to identify the significant geometric errors of a vertical three-axis milling machine center. Cheng et al. [16] introduced Sobol method into a vertical three-axis milling machine center to identify the geometric errors that most strongly affect the machining accuracy and successfully determined the vital geometric errors that contributed the most to the processing inaccuracy. Several optimization measures have been then adopted, and the improvement of machining accuracy verified the effectiveness of Sobol method.

This literature review demonstrates that the sensitivity analysis is very efficient to quantify the influence of the geometric errors on the error modeling; thus, sensitivity analysis has received lots of attention in the past time. The global sensitivity analysis method is suitable to discriminate the crucial geometric errors in the error modeling of a measuring machine or a processing machine tool. However, relatively few investigations have been performed for a 5axis measuring machine, and the influence of geometric errors on the measurement result is not well understood. To overcome this limitation, the present study employs Sobol method to perform the sensitivity analysis of geometric errors of a 5-axis measuring machine and to quantify the effect of the geometric error items on the measurement result of the 5-axis measuring machine.

In general, there are two approaches to significantly decrease the measurement error for a measuring machine: the first one is to completely eliminate the geometric errors of a measuring machine while the second one is to reasonably distribute and control the error level of each geometric error according to the sensitivity analysis result in order to equalize the effect of geometric errors on the measurement result. Obviously, the first approach is more difficult to achieve because the geometric errors are inevitably introduced in the assembly and manufacturing processes. In contrast, the second approach is a more feasible way and has been widely used in practical production, which requires to moderately reduce the error levels of some crucial geometric errors with a larger sensitivity index and to finally ensure all the sensitivity indices of geometric errors equivalent as possible.

The present study aims to achieve the error distribution of the prototype of a 5-axis measuring machine based on sensitivity analysis of geometric errors. The measurement error modeling is first obtained by homogeneous coordinate transformation, Sobol method is then employed to determine the crucial geometric errors that contribute strong influence to the measurement result of a 5-axis measuring machine, and the implementation solution of error distribution in the design stage is finally discussed in detail. The homogeneous coordinate transformation method is briefly reviewed in Section 2, and the measurement error modeling of a 5-axis measuring machine is obtained. Section 3 introduces the Sobol sensitivity analysis method and its working principle. Section 4 discusses the sensitivity analysis result in detail and proposes the error distribution measures. Finally, Section 5 provides significant conclusions.

\section{Measurement Error Modeling of the 5-Axis Measuring Machine}

2.1. The Description of the 5-Axis Measuring Machine. A 5axis measuring machine is employed to measure a workpiece designed for a special purpose. The principal feature of the workpiece is a sphere with radius $R_{\mathrm{s}} 25 \mathrm{~mm}$, and it is supported by a cylinder with radius $R_{\mathrm{d}} 10 \mathrm{~mm}$ in the $Z$ positive direction, as shown in Figure 1. Besides, five planes 


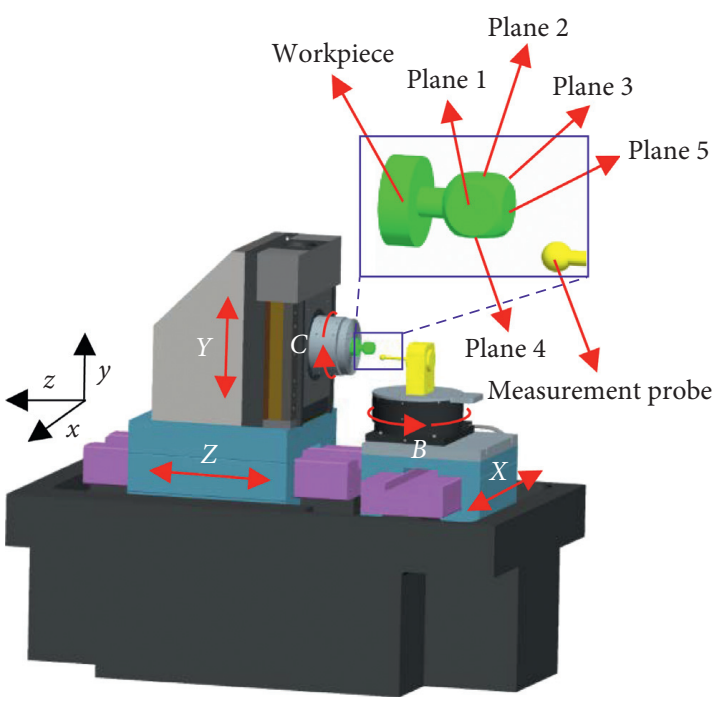

FIgURE 1: The schematic diagram of the 5-axis measuring machine.

on the workpiece surface are parallel to the coordinate system, and the distances of these planes to the sphere center are $18 \mathrm{~mm}$ (plane 1 in the $X$ positive direction), $20 \mathrm{~mm}$ (plane 3 in the $X$ negative direction), $19 \mathrm{~mm}$ (plane 2 in the $Y$ positive direction), $20 \mathrm{~mm}$ (plane 4 in the $Y$ negative direction), and $18.5 \mathrm{~mm}$ (plane 5 in the $Z$ negative direction), respectively. The required technical parameters are as follows: the surface roughness is $R_{\mathrm{a}} \leq 50 \mathrm{~nm}$, the sphericity of the main body is $\leq 2 \mu \mathrm{m}$, the flatness of these five planes is $\leq 1 \mu \mathrm{m}$, and the perpendicularity and parallelism between planes are $\leq 2 \mu \mathrm{m}$. These main measurement parameters are strictly required during the measuring process.

Correspondingly, the proposed schematic of the 5-axis measuring machine is shown in Figure 1. The 5-axis measuring machine composes of two rotational axes (the $B$ axis and $C$-axis) and three translational axes (the $X$-axis, $Y$ axis, and $Z$-axis, respectively). The coordinate system is a right-hand rectangular system, and the nomenclature of each axis is defined in accordance with the rules mentioned in ISO 841 [17].

The $X$-axis and $Z$-axis are horizontally fastened to the base of the 5 -axis measuring machine, the operation reference line of the $X$-axis is required to be perpendicular to that of the $Z$-axis, and the $Y$-axis is mounted on the top of the $Z$-axis and carries the $C$-axis in which the $Y$-axis moves along the vertical direction. The $B$-axis is installed on the $X$ axis, and the axis of rotation of the $B$-axis is parallel to the operation reference line of the $Y$-axis. Analogously, the axis of rotation of the $C$-axis is parallel to the operation reference line of the $Z$-axis. A vacuum chuck is mounted to the $C$-axis to support the workpiece while the measurement probe holder is supported by the $B$-axis. Both $B$-axis and $C$-axis serve as the angular servo axis.

The 5-axis measuring machine composes of eight-part rigid bodies [18], as shown in Figure 2. The base of the 5-axis measuring machine is selected as the zeroth-order body, and the remaining adjacent bodies are numbered in sequence; thus, the $Z$-axis, $Y$-axis, $C$-axis, workpiece, $X$-axis, $B$-axis,

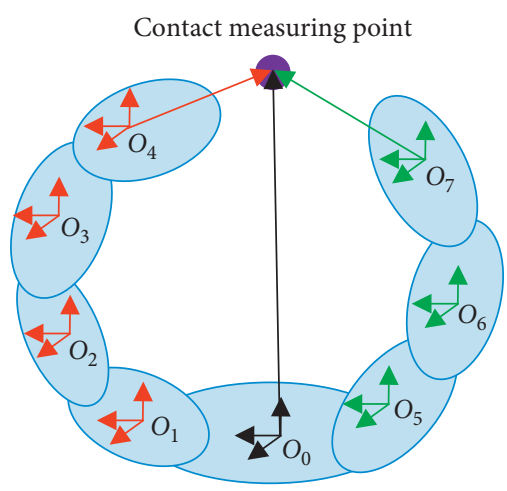

FIgURE 2: A topological schematic of the 5-axis measuring machine (0: machine base; 1 : $Z$-axis; 2 : $Y$-axis; 3 : $C$-axis; 4 : workpiece; $5: X$ axis; 6: $B$-axis; 7: measurement probe).

and the measurement probe are represented by rigid body 1 to 7 in succession, respectively. There exist two branches in the 5-axis measuring machine: the first branch (workpiece branch) comprises the $Z$-axis, $Y$-axis, $C$-axis, workpiece, and the machine base while the second branch (measurement probe branch) comprises the measurement probe, $B$-axis, $X$ axis, and the machine base; these two branches are connected by the machine base.

\subsection{Geometric Errors and Characteristic Matrices. Geometric} errors of machine tools are classified into position-dependent geometric errors and position-independent geometric errors $[9,10]$.

Position-dependent geometric errors are defined as the error motion caused by the linear movement or rotational movement of each axis. A kinematic axis introduces six geometric errors comprising linear and angular errors $[19,20]$. The six geometric errors of each translational axis ( $X$-axis, $Y$-axis, and $Z$-axis) are a single positioning error, two straightness errors, and three angular errors (pitch, yaw, and roll). Similarly, the six geometric errors of each rotational axis ( $B$-axis and $C$-axis) include three linear error components (a single axial error and two radial errors) and three angular errors (two tilt errors and an angular positioning error).

Position-independent geometric errors, also called location errors, are used to describe the reference lines of the axes of motion, including position errors, orientation errors, and zero position errors. For a translational axis, there exist three position-independent geometric errors, i.e., two orientation errors and the zero position error. For a rotational axis, there exist five position-independent geometric errors, i.e., two position errors, two orientation errors, and the zero angular position error (in analogy to the zero position error of a translational axis) [21-25].

Therefore, there exist 49 geometric errors (30 positionindependent geometric errors and 19 position-independent geometric errors) in a 5-axis measuring machine in total. Parts of these 19 position-independent geometric errors can be appropriately simplified and ignored. The zero position error can be set to zero [26]. Additionally, the position errors and some orientation errors can be 
negligible in measurement error modeling because of the choice of the coordinate system and reference position, and there remain only seven orientation errors, which refer to the volumetric squareness errors among the five axes [7]. It means that the location errors can be ignored when the squareness errors are fully considered; therefore, it can be considered that a 5-axis measuring machine totally introduces 37 geometric error items; this simplification will not affect the analysis result and is generally accepted in the field of machine tool $[7,18,27]$.

The influence of 37 geometric errors on the measurement result for a 5-axis measuring machine is investigated in this study, and the label from $h_{1}$ to $h_{37}$ is successively employed to stand for all 37 geometric errors for convenience, as listed in Table 1.

A $4 \times 4$ homogeneous transformation matrix is very powerful to express the position and posture between the two adjacent bodies in the 5-axis measuring machine. Usually, the transformation matrix between the $i$ th-order body and the $j$ th-order body is expressed as

$$
{ }_{i}^{j} T={ }_{i}^{j} T_{p i}^{j} T_{p e i}{ }^{j} T_{s i}^{j} T_{s e} \text {, }
$$

where ${ }_{i}^{j} T_{p}$ represents a position transformation matrix; ${ }_{i}^{j} T_{p e}$ represents a position error transformation matrix; ${ }_{i}^{j} T_{s}$ represents a motion transformation matrix; and ${ }_{i}^{j} T_{s e}$ represents a motion error transformation matrix.

Correspondingly, the characteristic matrices of the adjacent bodies for the 5-axis measuring machine are given in Tables 2 and 3. $p_{i j x}, p_{i j y}$, and $p_{i j z}$ in position transformation matrix ${ }_{i}^{j} T_{p}$ are the position coordinates of a rigid body $i$ in the coordinates of its adjacent high-order rigid body $j$, and the corresponding position coordinates of the 5-axis measuring machine are listed in Table 4.

2.3. Measurement Error Modeling. The measuring process can be regarded as the motion of the contact measuring point of measure probe within the workpiece coordinate system. Under the ideal situation without the influence of geometric errors, the measurement result is in accordance with the ideal trace of the workpiece.

The ideal contact measuring point in the workpiece coordinate could be written as

$$
\begin{aligned}
P_{w_{\text {ideal }}}= & {\left[\left({ }_{0}^{1} T_{p 0} T_{s}\right)\left({ }_{1}^{2} T_{p 1}{ }^{2} T_{s}\right)\left({ }_{2}^{3} T_{p 2}{ }^{3} T_{s}\right)\left({ }_{3}^{4} T_{p 3} T_{s}\right)\right]^{-1} } \\
& \cdot\left({ }_{5}^{0} T_{p 5} T_{s}\right)\left({ }_{5}^{6} T_{p 5} T_{s}\right)\left({ }_{6}^{7} T_{p 6}{ }^{7} T_{s}\right) P_{m},
\end{aligned}
$$

where $P_{w}=\left[P_{w x}, P_{w y}, P_{w z}, 1\right]^{\mathrm{T}}$ is the position of the contact measuring point in the workpiece coordinate while $P_{m}=$ $\left[P_{m x}, P_{m y}, P_{m z}, 1\right]^{\mathrm{T}}$ is the position of the contact measuring point in the measurement probe coordinate.

However, the 5-axis measuring machine unavoidably operates with the effect of 37 geometric errors; these geometric errors will cause the actual trajectory between the probe and workpiece deviated from the ideal one; the actual measurement result is inaccurate relative to the nominal one, and the difference is defined as the measurement error. Thus, the actual measurement result can be regarded as a superposition of the ideal measurement result of a workpiece and the measurement error caused by geometric errors.

Therefore, the actual contact measuring point in the workpiece coordinate can be written as

$$
P_{w_{-} \text {actual }}=\left[{ }_{0}^{1} T_{1}^{2} T_{2}^{3} T\right]^{-1}\left[{ }_{0}^{4} T_{4}^{5} T\right] P_{m} .
$$

The measurement error modeling caused by geometric errors can be written as

$$
E=\left[E_{x}, E_{y}, E_{z}, 1\right]^{\mathrm{T}}=P_{w_{-} \text {actual }}-P_{w_{-} \text {ideal }},
$$

where $E_{x}, E_{y}$, and $E_{z}$ are the components of measurement error $E$ along the $X, Y$, and $Z$ directions, respectively. According to the labels $h=\left[h_{1}, h_{2}, h_{3}, \ldots, h_{37}\right]$ listed in Table 1, equation (4) can be given as

$$
E=\left[E_{x}(h), E_{y}(h), E_{z}(h), 1\right]^{\mathrm{T}} .
$$

The scale of comprehensive error modeling can be expressed as

$$
Y=E=\sqrt{E_{x}(h)^{2}+E_{y}(h)^{2}+E_{z}(h)^{2}}
$$

To improve the accuracy and reliability of the measurement result, the measurement error caused by geometric errors is supposed to be reduced or removed, which directly requires decreasing or eliminating the geometric error as possible. In fact, geometric errors cannot be completely eradicated; it means that the 5-axis measuring machine inevitably operates under geometric errors; thus, the measurement error always exists. The relationship between the measurement error and geometric errors is not well understood; it is of great significance to reveal the influence of geometric errors on the measurement result. Sobol method is adopted to qualify the influence using the sensitivity index, which is beneficial to achieve the error distribution of a 5axis measuring machine.

\section{Global Sensitivity Analysis Method}

3.1. Basic Principle of Sobol Method. Sobol method has been one of the most effective global sensitivity analysis methods over the past several decades. Compared with other methods, Sobol method is of the advantages of fast calculation, high efficiency, and easy application.

Sobol method calculates the sensitivity index of the input parameter using the ratio of the variance of the input parameter to the total variance of the error model, in which the quasi-Monte Carlo sampling method is employed to improve the convergence and calculation accuracy. Sobol method can perform the sensitivity analysis of a mathematical model with up to 50 input parameters no matter whether the model was linear or nonlinear. One of the most outstanding advantages of Sobol method is that it is capable of simultaneously evaluating the first-order sensitivity index and the high-order sensitivity index, which are helpful to reveal the interaction effect between or among the input parameters. Therefore, Sobol method can be applied to quantify the influence of geometric errors on the measurement result for a 5-axis measuring machine. 
TABLE 1: Geometric errors of the 5-axis measuring machine.

\begin{tabular}{lcc}
\hline Axis & Error components* & Error labels \\
\hline$Z$-axis & $\delta_{z x}, \delta_{z y}, \delta_{z z}, \theta_{z x}, \theta_{z y}, \theta_{z z}$ & $h_{1}, h_{2}, h_{3}, h_{4}, h_{5}, h_{6}$ \\
$Y$-axis & $\delta_{y x}, \delta_{y y}, \delta_{y z}, \theta_{y x}, \theta_{y y}, \theta_{y z}$ & $h_{7}, h_{8}, h_{9}, h_{10}, h_{11}, h_{12}$ \\
$C$-axis & $\delta_{c x}, \delta_{c y}, \delta_{c z}, \theta_{c x}, \theta_{c y}, \theta_{c z}$ & $h_{15}, h_{16}, h_{17}, h_{18}, h_{19}, h_{20}$ \\
$X$-axis & $\delta_{x x}, \delta_{x y}, \delta_{x z}, \theta_{x x}, \theta_{x y}, \theta_{x z}$ & $h_{23}, h_{24}, h_{25}, h_{26}, h_{27}, h_{28}$ \\
$B$-axis & $\delta_{b x}, \delta_{b y}, \delta_{b z}, \theta_{b x}, \theta_{b y}, \theta_{b z}$ & $h_{30}, h_{31}, h_{32}, h_{33}, h_{34}, h_{35}$ \\
Squareness errors & $\beta_{y z}, \beta_{y x}, \beta_{c x}, \beta_{c y}, \beta_{z x}, \beta_{b x}, \beta_{b z}$ & $h_{13}, h_{14}, h_{21}, h_{22}, h_{29}, h_{36}, h_{37}$ \\
\hline
\end{tabular}

* where $\delta_{m n}$ delegates the linear errors, $\theta_{m n}$ delegates the angular errors, the left subscript $m$ delegates the kinematic axis while the right subscript $n$ delegates the direction of linear error or the rotational axis of the angular error, and $\beta_{m n}$ delegates the squareness errors of $m$ axis to $n$ axis, respectively.

TABLE 2: Position and position error transformation matrices of the 5-axis measuring machine (as referred to in Figure 2), where I represents the identity matrix.

\begin{tabular}{|c|c|c|}
\hline Adjacent bodies & Position transformation matrix ${ }_{i}^{j} T_{p}$ & Position error transformation matrix ${ }_{i}^{j} T_{p e}$ \\
\hline 0-1 (Z-axis) & {$\left[\begin{array}{cccc}1 & 0 & 0 & p_{01 x} \\
0 & 1 & 0 & p_{01 y} \\
0 & 0 & 1 & p_{01 z} \\
0 & 0 & 0 & 1\end{array}\right]$} & $\mathbf{I}_{4 \times 4}$ \\
\hline $1-2$ (Y-axis) & {$\left[\begin{array}{cccc}1 & 0 & 0 & p_{12 x} \\
0 & 1 & 0 & p_{12 y} \\
0 & 0 & 1 & p_{12 z} \\
0 & 0 & 0 & 1\end{array}\right]$} & {$\left[\begin{array}{cccc}1 & -\beta_{y x} & 0 & 0 \\
\beta_{y x} & 1 & -\beta_{y z} & 0 \\
0 & \beta_{y z} & 1 & 0 \\
0 & 0 & 0 & 1\end{array}\right]$} \\
\hline 2-3 (C-axis) & {$\left[\begin{array}{cccc}1 & 0 & 0 & p_{23 x} \\
0 & 1 & 0 & p_{23 y} \\
0 & 0 & 1 & p_{23 z} \\
0 & 0 & 0 & 1\end{array}\right]$} & {$\left[\begin{array}{cccc}1 & 0 & \beta_{c x} & 0 \\
0 & 1 & -\beta_{c y} & 0 \\
-\beta_{c x} & \beta_{c y} & 1 & 0 \\
0 & 0 & 0 & 1\end{array}\right]$} \\
\hline 3-4 (workpiece) & {$\left[\begin{array}{cccc}1 & 0 & 0 & p_{34 x} \\
0 & 1 & 0 & p_{34 y} \\
0 & 0 & 1 & p_{34 z} \\
0 & 0 & 0 & 1\end{array}\right]$} & $\mathbf{I}_{4 \times 4}$ \\
\hline $0-5$ (X-axis) & {$\left[\begin{array}{cccc}1 & 0 & 0 & p_{05 x} \\
0 & 1 & 0 & p_{05 y} \\
0 & 0 & 1 & p_{05 z} \\
0 & 0 & 0 & 1\end{array}\right]$} & {$\left[\begin{array}{cccc}1 & 0 & \beta_{z x} & 0 \\
0 & 1 & 0 & 0 \\
-\beta_{z x} & 0 & 1 & 0 \\
0 & 0 & 0 & 1\end{array}\right]$} \\
\hline 5-6 (B-axis) & {$\left[\begin{array}{cccc}1 & 0 & 0 & p_{56 x} \\
0 & 1 & 0 & p_{56 y} \\
0 & 0 & 1 & p_{56 z} \\
0 & 0 & 0 & 1\end{array}\right]$} & {$\left[\begin{array}{cccc}1 & -\beta_{b x} & 0 & 0 \\
\beta_{b x} & 1 & -\beta_{b z} & 0 \\
0 & \beta_{b z} & 1 & 0 \\
0 & 0 & 0 & 1\end{array}\right]$} \\
\hline 6-7 (probe) & {$\left[\begin{array}{cccc}1 & 0 & 0 & p_{67 x} \\
0 & 1 & 0 & p_{67 y} \\
0 & 0 & 1 & p_{67 z} \\
0 & 0 & 0 & 1\end{array}\right]$} & $\mathbf{I}_{4 \times 4}$ \\
\hline
\end{tabular}

The basic flow of sensitivity analysis using Sobol method is shown in Figure 3 in detail.

(1) Generate two random sampling matrices $\mathbf{A}_{k \times n}$ and $\mathbf{B}_{k \times n}$ based on the Sobol sequence and the probability distribution of the geometric errors, which is required as a pre-requisite to evaluate the sensitivity index, the Sobol sequences method is freely available in MATLAB software. The matrices $\mathbf{A}_{k \times n}$ and $\mathbf{B}_{k \times n}$ both contain $k$ rows and $n$ columns; $k$ is the sampling number of each geometric error and $n$ corresponds to the number of geometric errors. Matrix $\mathbf{A}_{B}^{(i)}$ is defined that only the $i$-th column of matrix $\mathbf{A}_{k \times n}$ is replaced by the $i$-th column of matrix $\mathbf{B}_{k \times n}$, and all the remaining columns of matrix $\mathbf{A}_{k \times n}$ remain unchanged [12].

(2) Construct a mathematical model that accurately represents the actual problem, and the model is the basis of the sensitivity analysis. The mathematical model in this study is the measurement error 
TABLE 3: Motion and motion error transformation matrices of the 5-axis measuring machine (as referred to in Figure 2), where I represents the identity matrix.

\begin{tabular}{|c|c|c|}
\hline Adjacent bodies & Motion transformation matrix ${ }_{i}^{j} T_{s}$ & Motion error transformation matrix ${ }_{i}^{j} T_{s e}$ \\
\hline 0-1 (Z-axis) & {$\left[\begin{array}{llll}1 & 0 & 0 & 0 \\
0 & 1 & 0 & 0 \\
0 & 0 & 1 & Z \\
0 & 0 & 0 & 1\end{array}\right]$} & {$\left[\begin{array}{cccc}1 & -\theta_{z z} & \theta_{z y} & \delta_{z x} \\
\theta_{z z} & 1 & -\theta_{z x} & \delta_{z y} \\
-\theta_{z y} & \theta_{z x} & 1 & \delta_{z z} \\
0 & 0 & 0 & 1\end{array}\right]$} \\
\hline 1-2 (Y-axis) & {$\left[\begin{array}{llll}1 & 0 & 0 & 0 \\
0 & 1 & 0 & Y \\
0 & 0 & 1 & 0 \\
0 & 0 & 0 & 1\end{array}\right]$} & {$\left[\begin{array}{cccc}1 & -\theta_{y z} & \theta_{y y} & \delta_{y x} \\
\theta_{y z} & 1 & -\theta_{y x} & \delta_{y y} \\
-\theta_{y y} & \theta_{y x} & 1 & \delta_{y z} \\
0 & 0 & 0 & 1\end{array}\right]$} \\
\hline $2-3$ (C-axis) & {$\left[\begin{array}{cccc}\cos C & -\sin C & 0 & 0 \\
\sin C & \cos C & 0 & 0 \\
0 & 0 & 1 & 0 \\
0 & 0 & 0 & 1\end{array}\right]$} & {$\left[\begin{array}{cccc}1 & -\theta_{c z} & \theta_{c y} & \delta_{c x} \\
\theta_{c z} & 1 & -\theta_{c x} & \delta_{c y} \\
-\theta_{c y} & \theta_{c x} & 1 & \delta_{c z} \\
0 & 0 & 0 & 1\end{array}\right]$} \\
\hline 3-4 (workpiece) & $\mathbf{I}_{4 \times 4}$ & $\mathbf{I}_{4 \times 4}$ \\
\hline $0-5$ ( $X$-axis $)$ & {$\left[\begin{array}{llll}1 & 0 & 0 & X \\
0 & 1 & 0 & 0 \\
0 & 0 & 1 & 0 \\
0 & 0 & 0 & 1\end{array}\right]$} & {$\left[\begin{array}{cccc}1 & -\theta_{x z} & \theta_{x y} & \delta_{x x} \\
\theta_{x z} & 1 & -\theta_{x x} & \delta_{x y} \\
-\theta_{x y} & \theta_{x x} & 1 & \delta_{x z} \\
0 & 0 & 0 & 1\end{array}\right]$} \\
\hline 5-6 (B-axis) & {$\left[\begin{array}{cccc}\cos B & 0 & \sin B & 0 \\
0 & 1 & 0 & 0 \\
-\sin B & 0 & \cos B & 0 \\
0 & 0 & 0 & 1\end{array}\right]$} & {$\left[\begin{array}{cccc}1 & -\theta_{b z} & \theta_{b y} & \delta_{b x} \\
\theta_{b z} & 1 & -\theta_{b x} & \delta_{b y} \\
-\theta_{b y} & \theta_{b x} & 1 & \delta_{b z} \\
0 & 0 & 0 & 1\end{array}\right]$} \\
\hline 6-7 (probe) & $\mathbf{I}_{4 \times 4}$ & $\mathbf{I}_{4 \times 4}$ \\
\hline
\end{tabular}

TABLE 4: The position coordinates between body $i$ and body $j$.

\begin{tabular}{lccc}
\hline & $p_{i j x}$ & $p_{i j y}$ & $p_{i j z}$ \\
\hline$i=0, j=1$ (Z-axis) & 0 & 260 & 240 \\
$i=1, j=2$ (Y-axis) & 0 & 275 & -240 \\
$i=2, j=3$ (C-axis) & 0 & 0 & -175 \\
$i=3, j=4$ (workpiece) & 0 & 0 & -35 \\
$i=0, j=5$ (X-axis) & 0 & 180 & -545 \\
$i=5, j=6$ (B-axis) & 0 & 205 & 85.8 \\
$i=6, j=7$ (probe) & 0 & 82 & 158.2 \\
\hline
\end{tabular}

modeling $Y=f(h)$ for $h=\left(h_{1}, h_{2}, \ldots, h_{n}\right)$, and $n$ is the total number of geometric error items in a 5 -axis measuring machine.

(3) Substitute the input matrices $\mathbf{A}_{k \times n}, \mathbf{B}_{k \times n}$, and $\mathbf{A}_{B}^{(i)}$, respectively, into the measurement error modeling $Y=f(h)$. This calculation process runs for $k \times(n+2)$ times and finally obtain $f(\mathbf{A}), f(B)$, and $f\left(\mathbf{A}_{B}^{(i)}\right)$. The simulation calculation is carried out in MATLAB software.

(4) Calculate the variances according to the variance estimator.

(5) Evaluate the sensitivity indices by the variance ratio.

According to the thought of Sobol method [28, 29], the Sobol model suitable for a 5 -axis measuring machine can be described by $Y=f(h)$, in which $h=\left(h_{1}, h_{2}, \ldots, h_{37}\right)$ represents 37 geometric error items in a 5-axis measuring machine. According to the uniqueness of analysis of variance representation $[30,31]$, the measurement error modeling function $Y=f(h)$ can be further expressed by the increments series

$$
Y=Y_{0}+\sum_{i=1}^{n} Y_{i}+\sum_{i=1}^{n} \sum_{j>i}^{n} Y_{i j}+\cdots+Y_{1,2, \ldots, n},
$$

where $Y_{0}$ is the expected value of the model for input variables, $Y_{i}=Y_{i}\left(h_{i}\right), Y_{i j}=Y_{i j}\left(h_{i}, h_{j}\right)$, the similar regularity is suitable for the high-order items. The subitems in equation (7) are required to be orthogonal to each other. 


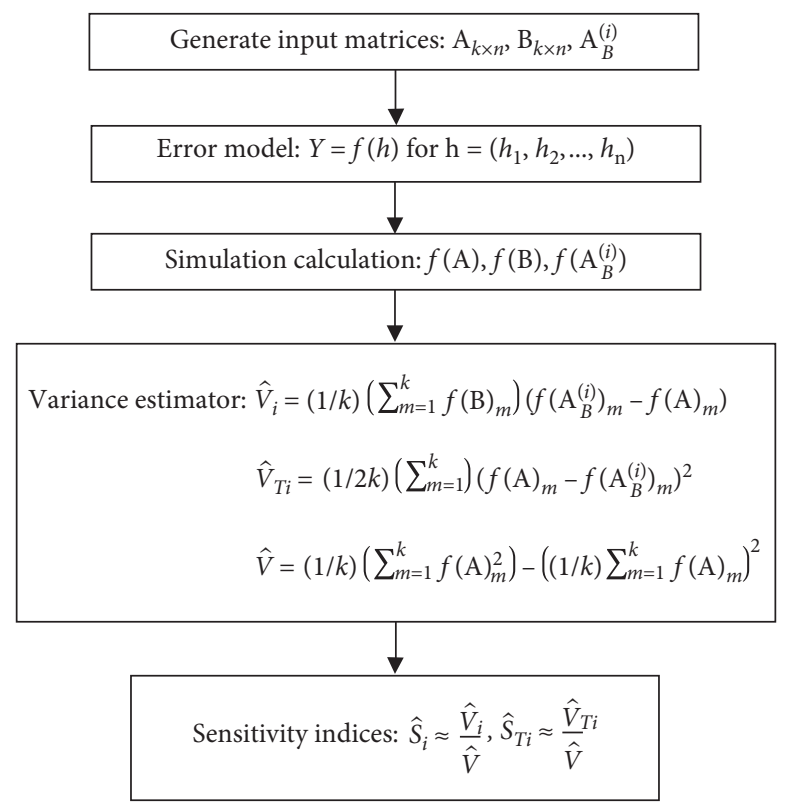

Figure 3: The flowchart of sensitivity analysis using Sobol method.

Based on the mathematical statistics, equation (7) can be expressed in the form of variance $V$ as

$$
V=\sum_{i=1}^{n} V_{i}+\sum_{i=1}^{n} \sum_{j>i}^{n} V_{i j}+\cdots+V_{1,2, \ldots, n}
$$

After the normalization of dividing by the variance $V$, equation (8) is transformed into

$$
\sum_{i=1}^{n} S_{i}+\sum_{i=1}^{n} \sum_{j>i}^{n} S_{i j}+\cdots+S_{1,2, \ldots, n}=1 .
$$

The variance ratio

$$
S_{i}=\frac{V_{i}}{V}
$$

is defined as the first-order global sensitivity index [12, 32]. $S_{i}$ evaluates the main influence of the geometric error $h_{i}$ on the measurement result, which augments with the increasing importance of the geometric error.

$S_{i j}$ accounts for the interaction effect between two inputs $h_{i}$ and $h_{j}$ when $i \neq j$, similarly to high-order terms. $S_{T i}$ is another commonly used sensitivity index, named the total sensitivity index, and it corresponds to the sum of the firstorder sensitivity index of $h_{i}$ and all the interaction indices including $h_{i}$ [33].

3.2. The Estimation Method of $S_{i}$. As mentioned above, Sobol method employs Sobol sequences to improve the efficiency of the estimators [34]. Two random sampling matrices $\mathbf{A}_{k \times n}$ and $\mathbf{B}_{k \times n}$ and one deduced matrix $\mathbf{A}_{B}^{(i)}$ are required for evaluating the sensitivity index. The commonly used estimators for $S_{i}$ and $S_{T i}$ are [29]

$$
\begin{aligned}
\widehat{V}_{i} & =\frac{1}{k} \sum_{m=1}^{k} f(\mathbf{B})_{m}\left(f\left(\mathbf{A}_{B}^{(i)}\right)-f(\mathbf{A})_{m}\right), \\
\widehat{V}_{T i} & =\frac{1}{2 k} \sum_{m=1}^{k}\left(f(\mathbf{A})_{m}-f\left(\mathbf{A}_{B}^{(i)}\right)_{m}\right)^{2}, \\
\widehat{V} & =\frac{1}{k} \sum_{m=1}^{k} f(\mathbf{A})_{m}^{2}-\left[\frac{1}{k} \sum_{m=1}^{k} f(\mathbf{A})_{m}\right]^{2},
\end{aligned}
$$

where $(\mathbf{A})_{m}$ and $(\mathrm{B})_{m}$ denote the $m$-th column of matrices $\mathbf{A}$ and $\mathbf{B}$, respectively.

3.3. The Workspace and Geometric Error Intervals. As discussed, the prototype of a 5-axis measuring machine is designed as shown in Figure 1; the stroke of $X$-axis, $Y$-axis, and $Z$-axis is, respectively, $\pm 75 \mathrm{~mm}, \pm 50 \mathrm{~mm}$, and $\pm 75 \mathrm{~mm}$, and the rotation range of $C$-axis is $360^{\circ}$ while that of $B$-axis is from $-120^{\circ}$ to $+120^{\circ}$. Therefore, there exist four body diagonals in the whole workspace of $150 \mathrm{~mm} \times 100 \mathrm{~mm} \times 150 \mathrm{~mm}$, as shown in Figure 4; each diagonal of the workspace is evenly divided into six segments before conducting the sensitivity analysis. The five points along each diagonal near the workspace center are selected as test points because these points are located in the most frequently used coordinate range of the 5axis measuring machine, and four body diagonals generate 17 test points in total in the workspace, performing the sensitivity analysis at these points will provide more meaningful results. It is noted that $B$-axis is parallel to $Y$-axis; therefore, the rotation angle of $B$-axis is arranged synchronously with the location coordinate of $Y$-axis in the process of sensitivity analysis, similarly $C$-axis to $Z$-axis. Correspondingly, the coordinates of each test point are listed in Table 5.

Based on the previous experience in developing an ultraprecision machine tool, the error levels of geometric error 


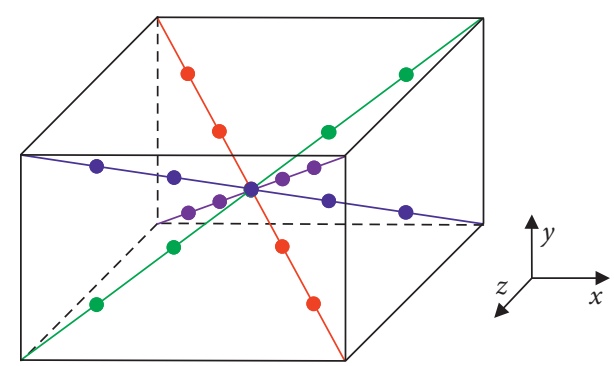

FIgURE 4: The test points in the workspace.

TABLE 5: The coordinates of each test point in the workspace.

\begin{tabular}{lccccc}
\hline Test point & $X(\mathrm{~mm})$ & $Y(\mathrm{~mm})$ & $Z(\mathrm{~mm})$ & $B$ (degree) & $C$ (degree) \\
\hline 1 & -50 & -33.33 & -50 & -80 & -120 \\
2 & -50 & -33.33 & 50 & -80 & 120 \\
3 & -50 & 33.33 & -50 & 80 & -120 \\
4 & -50 & 33.33 & 50 & 80 & 120 \\
5 & -25 & -16.67 & -25 & -40 & -60 \\
6 & -25 & -16.67 & 25 & -40 & 60 \\
7 & -25 & 16.67 & -25 & 40 & -60 \\
8 & -25 & 16.67 & 25 & 40 & 60 \\
9 & 0 & 0 & 0 & 0 & 0 \\
10 & 25 & -16.67 & -25 & -40 & -60 \\
11 & 25 & -16.67 & 25 & -40 & 60 \\
12 & 25 & 16.67 & -25 & 40 & -60 \\
13 & 25 & 16.67 & 25 & 40 & 60 \\
14 & 50 & -33.33 & -50 & -80 & -120 \\
15 & 50 & -33.33 & 50 & -80 & 120 \\
16 & 50 & 33.33 & -50 & 80 & -120 \\
17 & 50 & 33.33 & 50 & 80 & 120 \\
\hline
\end{tabular}

TABLE 6: The statistical interval of geometric errors.

\begin{tabular}{lc}
\hline Geometric error item & Error level \\
\hline$h_{3}, h_{8}, h_{23}$ & $\pm 1 \mu \mathrm{m} / 100 \mathrm{~mm}$ \\
$h_{1}, h_{2}, h_{7}, h_{9}, h_{15}, h_{16}, h_{17}, h_{24}, h_{25}, h_{30}, h_{31}, h_{32}$ & $\pm 0.5 \mu \mathrm{m} / 100 \mathrm{~mm}$ \\
$h_{4}, h_{5}, h_{6}, h_{10}, h_{11}, h_{12}, h_{13}, h_{14}, h_{18}, h_{19}, h_{20}, h_{21}$, & \\
$h_{22}, h_{26}, h_{27}, h_{28}, h_{29}, h_{33}, h_{34}, h_{35}, h_{36}, h_{37}$ & $\pm 5^{\prime \prime}$ \\
\hline
\end{tabular}

for the prototype of a 5-axis measuring machine are summarized and listed in Table 6, all the positioning errors of translational axes are $\pm 1 \mu \mathrm{m} / 100 \mathrm{~mm}$, all the linear errors except the positioning errors are $\pm 0.5 \mu \mathrm{m} / 100 \mathrm{~mm}$, and all the angular errors and squareness errors are $\pm 5^{\prime \prime}$.

A Monte Carlo simulation of sensitivity analysis could involve thousands or tens of thousands of iteration calculations. With the increasing sampling number of each geometric error, the simulation accuracy and time consuming could be simultaneously increased. For the objective of utilizing the lower sampling number to attain the same simulation accuracy in a shorter time, it is necessary to balance the relationship between the sampling number and time consuming. 10000 samplings are sufficient for each geometric error under the premise of ensuring the simulation accuracy [16]; thus, two independent $10000 \times 37$ sampling matrixes $\mathbf{A}$ and $\mathbf{B}$ are constructed by Sobol sequences for the simulations, and the sensitivity index of geometric errors at each test point can be obtained by performing Sobol method; the sensitivity index is finally calculated using the average method. The sensitivity analysis result will be discussed in Section 4 .

\section{Results and Discussion}

It is commonly accepted that the first-order sensitivity index $S_{i}$ represents the main influence of the geometric error $h_{i}$ on the measurement result. It is proved that $S_{i}$ is capable of adequately representing $S_{T i}$ when the error levels of geometric errors are relatively small [35], which means that the interaction between any two arbitrary geometric errors can be ignored in the sensitivity analysis process, and this simplification can still provide valuable guidance without affecting the analysis result. Therefore, only $S_{i}$ is henceforth discussed in the study.

The sensitivity analysis was conducted at each test point which is listed in Table 5 to investigate the influence of geometric errors on the comprehensive error modeling and its component along the $X, Y$, and $Z$ directions. The corresponding results of the sensitivity analysis are subsequently discussed in detail.

As mentioned, the sum of all the first-order sensitivity indices is regarded as 1 when the higher-order sensitivity indices are neglected. There are 37 geometric errors in the 5axis measuring machine; thus, the sensitivity index 0.05 (approximately twice as the mean sensitivity index) is selected as the critical threshold to determine whether the geometric error provides a significant influence on the measurement result. If the sensitivity index $S_{i}$ of a geometric error $h_{i}$ is greater than 0.05 , the geometric error $h_{i}$ can be regarded as a crucial sensitive error, and the geometric errors whose sensitivity index is below 0.05 are hereby nonsensitive error items.

4.1. Sensitivity Analysis Result of Geometric Errors. The Xdirectional result of sensitivity index $S_{i}$ is displayed in Figure 5. It is obvious that $h_{5}$ and $h_{28}$ are the two outstanding geometric errors contributing enormously to the $X$-directional measurement error. Additionally, it is observed that the values of $S_{i}$ for $h_{6}, h_{11}, h_{27}$, and $h_{29}$ are greater than 0.05 ; thus these four geometric errors are important geometric errors. In addition, $h_{27}$ and $h_{29}$ have an equivalent degree of effect on the $X$-directional measurement error. The remaining geometric error items have little or no influence on the measurement result and are therefore not considered as crucial geometric errors. From these results, it is concluded that $h_{5}, h_{6}, h_{11}, h_{27}, h_{28}$, and $h_{29}$ are the crucial geometric errors that seriously impress the $X$-directional measurement error.

According to the labels listed in Table 1, these above six error items are $\theta_{x y}, \theta_{y y}, \theta_{z y}, \theta_{x z}, \theta_{z z}$, and $\beta_{z x}$, and it is noted that the crucial geometric errors influencing strongly the $X$ directional measurement error are angular and squareness errors, i.e., three angular errors $\left(\theta_{x y}, \theta_{y y}\right.$, and $\left.\theta_{z y}\right)$ along the $Y$ direction, respectively, attributed to the motion of $X$-axis, $Y$ axis, and $Z$-axis, two angular errors $\left(\theta_{x z}\right.$ and $\left.\theta_{z z}\right)$ along the $Z$ 


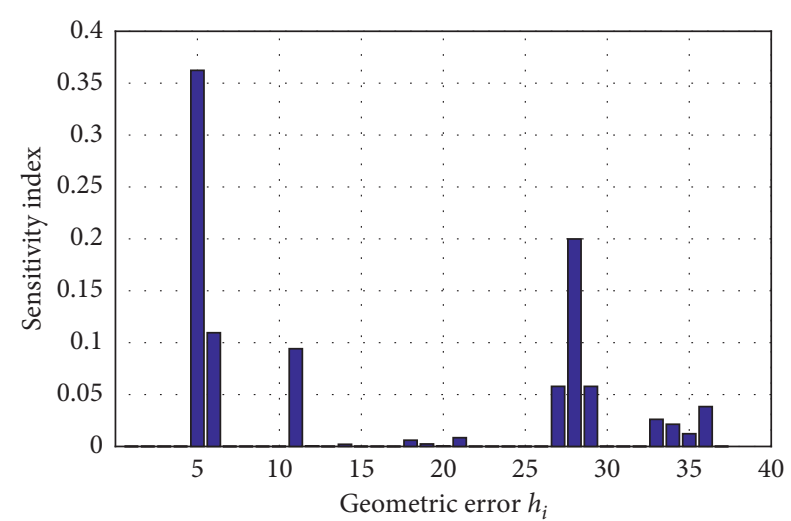

FIgURE 5: The $X$-directional sensitivity indices of geometric errors.

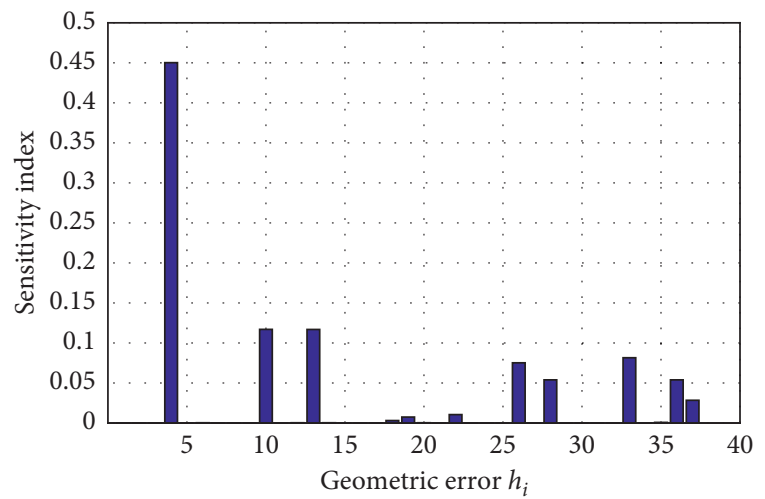

Figure 6: The $Y$-directional sensitivity indices of geometric errors.

direction, respectively, attributed to the motion of $X$-axis and $Z$-axis, and one squareness error $\left(\beta_{z x}\right)$ of $X$-axis to $Z$ axis.

As was conducted previous, the sensitivity analysis result along the $Y$ direction is shown in Figure 6, and $h_{4}$ is the most important factor affecting the $Y$-directional measurement error and contributes over $40 \%$ of the total sum of $S_{i}$. Besides, the sensitivity index $S_{i}$ of $h_{10}, h_{13}, h_{26}, h_{28}, h_{33}$, and $h_{36}$ is greater than 0.05 . Based on the above information, it can be reasonably concluded that $h_{4}, h_{10}, h_{13}, h_{26}, h_{28}, h_{33}$, and $h_{36}$ are the crucial geometric errors influencing the $Y$-directional measurement error, and $h_{4}$ plays the most important role in the $Y$-directional measurement error. Accordingly, $\theta_{x x}, \theta_{y x}, \theta_{z x}, \theta_{b x}, \beta_{y z}$, and $\beta_{b x}$ are the geometric errors that have a serious impact on the measurement result along the $Y$ direction, i.e., four angular errors $\left(\theta_{x x}, \theta_{y x}, \theta_{z x}\right.$, and $\theta_{b x}$ ) along the $X$ direction, respectively, attributed to the motion of $X$-axis, $Y$-axis, $Z$-axis, and $B$-axis, one angular error $\left(\theta_{x z}\right)$ along the $Z$ direction attributed to the motion of $X$-axis, and two squareness errors $\left(\beta_{y z}\right.$ and $\left.\beta_{b x}\right)$ of $Y$-axis to $Z$-axis and $B$-axis to $X$-axis. It is noted that the angular and squareness errors remained dominant comparing to the linear errors.

Figure 7 shows the sensitivity analysis result along the $Z$ direction, and $h_{26}$ is the most important geometric error with the sensitivity index 0.39 . In addition, $h_{4}$ is another critical geometric error with the sensitivity index greater

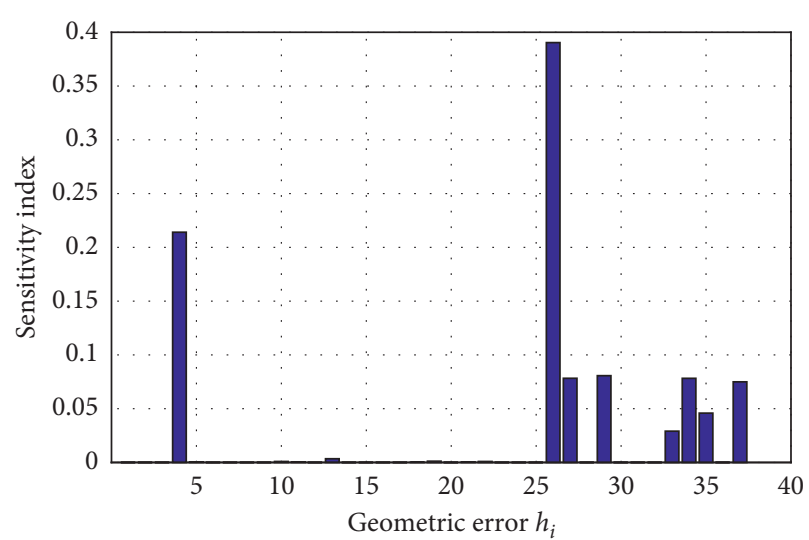

FIgURE 7: The Z-directional sensitivity indices of geometric errors.

than 0.20. $h_{27}, h_{29}, h_{34}$, and $h_{37}$ are considered as the critical geometric errors, and these geometric errors provide an equivalent degree of influence on the $Z$-directional measurement error. Therefore, $\theta_{x x}, \theta_{z x}, \theta_{x y}, \theta_{b y}, \beta_{z x}$, and $\beta_{b z}$ are the crucial geometric errors contributing serious effect on the measurement result along the $Z$ direction, i.e., two angular errors $\left(\theta_{x x}\right.$ and $\left.\theta_{z x}\right)$ along the $X$ direction, respectively, attributed to the motion of $X$-axis and $Z$-axis, two angular errors $\left(\theta_{x y}\right.$ and $\left.\theta_{b y}\right)$ along the $Y$ direction, respectively, attributed to the motion of $X$-axis and $B$-axis, and two squareness errors ( $\beta_{z x}$ and $\beta_{b z}$ ) of $Z$-axis to $X$-axis and $B$-axis to $Z$-axis.

The sensitivity indices of geometric errors with respect to comprehensive error modeling are investigated, and the corresponding result is given in Figure 8. It is obvious that each sensitivity index of $h_{4}, h_{5}, h_{6}, h_{11}, h_{26}, h_{27}, h_{28}$, and $h_{29}$ is greater than the critical threshold 0.05 , which leads to the conclusion that the comprehensive error modeling is sensitive to these geometric errors including $\theta_{x x}, \theta_{x y}, \theta_{x z}, \theta_{y y}, \theta_{z x}$, $\theta_{z y}, \theta_{z z}$, and $\beta_{z x}$.

$\theta_{x x}, \theta_{x y}$, and $\theta_{x z}$ represent the angular errors, respectively, along the $X, Y$, and $Z$ direction caused by the motion of $X$-axis, analogously for $\theta_{z x}, \theta_{z y}$, and $\theta_{z z}$.

$\theta_{y y}$ is the angular error along the $Y$ direction attributed to the motion of $Y$-axis, and $\beta_{z x}$ is the squareness error of $Z$-axis to $X$-axis.

From these results, it is noted that most of the crucial geometric errors in Figure 8 are consistent with those in Figures 5-7; this indicates that these crucial geometric errors, whose sensitivity index is greater than the critical threshold 0.05 , have a serious effect on both the comprehensive error modeling and its components along the $X, Y$, and $Z$ direction.

Therefore, the crucial geometric errors identified by sensitivity analysis are supposed to be fully considered in the design and manufacturing stages of the 5-axis measuring machine, which is helpful to reduce the influence of geometric error on the measurement result. Therefore, the error levels of these crucial geometric errors should be improved and distributed as possible in order to equalize their influence on the measurement result.

The geometric errors, as mentioned before, are difficult to completely eliminate because of the assembly and 


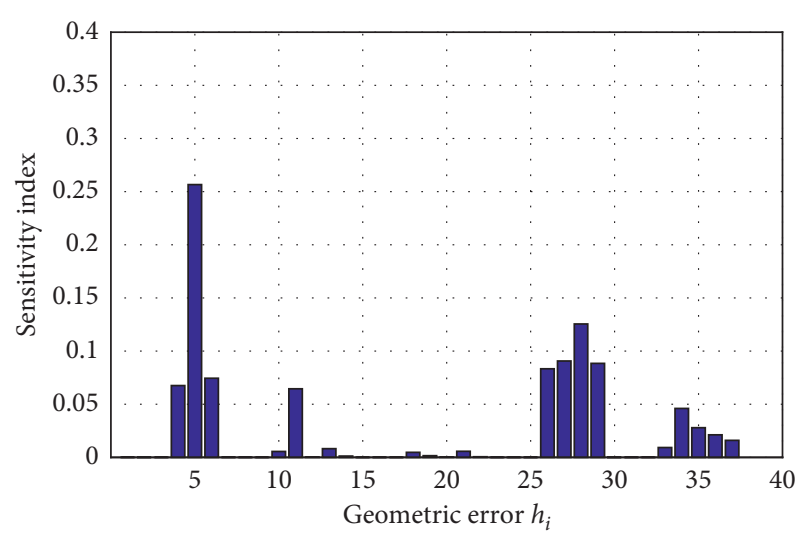

FIgURE 8: Sensitivity indices of geometric errors on comprehensive error modeling.

TABLE 7: The distributed error range of the crucial geometric errors.

\begin{tabular}{lcc}
\hline Geometric error item & Reduced by (\%) & Distributed error range \\
\hline$h_{10}, h_{13}, h_{27}, h_{34}$ & 20 & $\pm 4^{\prime \prime}$ \\
$h_{11}$ & 40 & $\pm 3^{\prime \prime}$ \\
$h_{6}, h_{29}$ & 50 & $\pm 2.5^{\prime \prime}$ \\
$h_{4}, h_{26}, h_{28}$ & 60 & $\pm 2^{\prime \prime}$ \\
$h_{5}$ & 70 & $\pm 1.5^{\prime \prime}$ \\
\hline
\end{tabular}

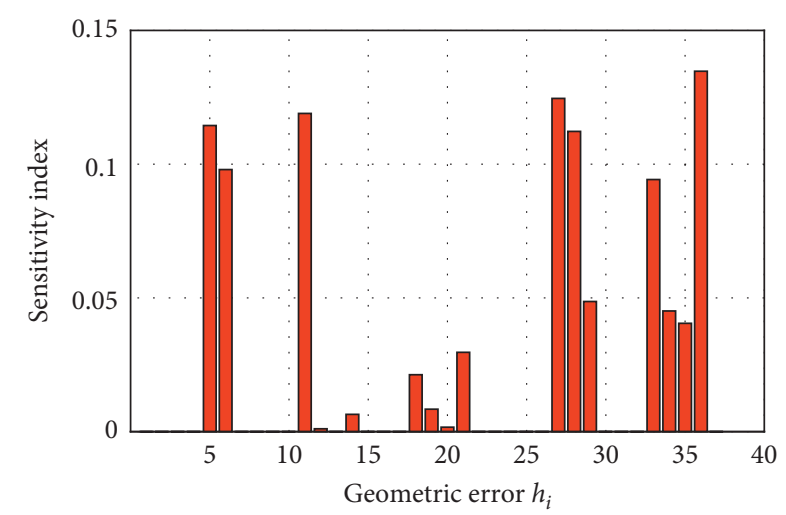

Figure 9: The $X$-directional sensitivity indices of geometric errors after error distribution.

manufacturing processes; it is a more feasible and reasonable approach of moderately distributing the error levels of the crucial geometric errors with larger sensitivity index in order to decrease the influence of some crucial geometric errors on measurement error. This method is employed in the study to improve the performance of the 5axis measuring machine, and the error levels of some crucial geometric errors are distributed and readjusted according to the sensitivity analysis results, as listed in Table 7; the corresponding reduction range is from $20 \%$ to $70 \%$, and the error levels of the remaining geometric errors remain constant.

After the distribution of geometric errors, the sensitivity analysis is re-executed to investigate the efficiency of the proposed error distribution method, and the result is available in the following subsection.

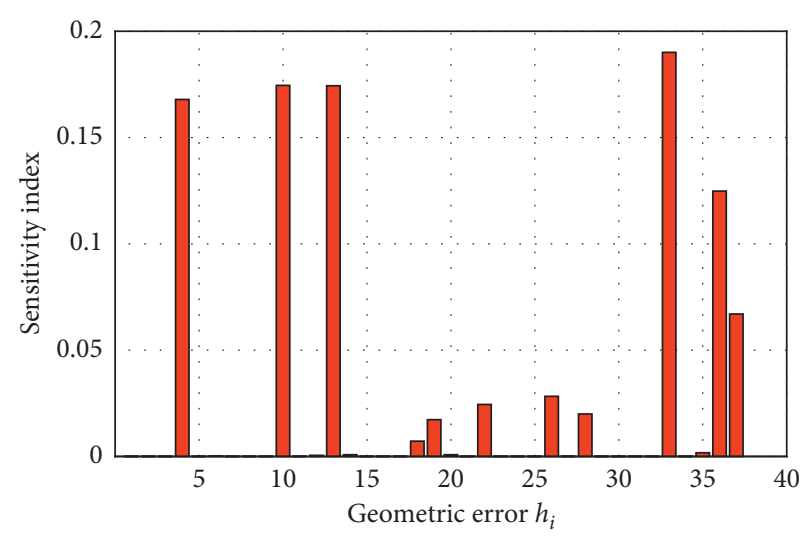

FIgURE 10: The $Y$-directional sensitivity indices of geometric errors after error distribution.

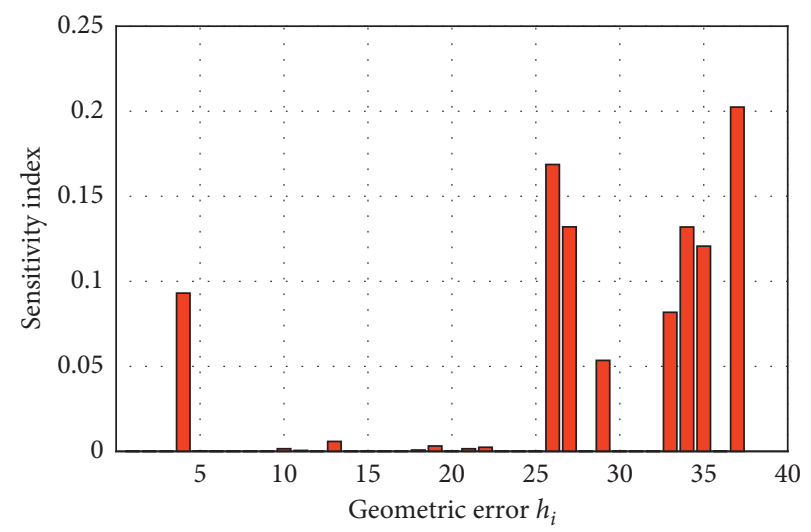

FIgURE 11: The Z-directional sensitivity indices of geometric errors after error distribution.

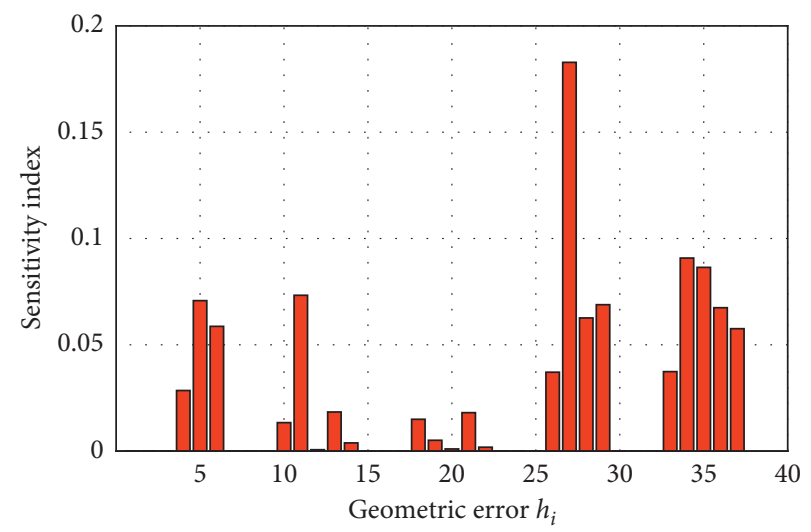

FIgURE 12: Sensitivity indices of geometric errors on comprehensive error modeling after error distribution.

4.2. Validation of the Effectiveness of Error Distribution. The sensitivity analysis is re-executed after the error levels are distributed, and Figures 9-12, respectively, show the sensitivity analysis result along the $X, Y$, and $Z$ directions and to comprehensive error modeling after error distribution.

Compared to Figure 5, it is obviously observed in Figure 9 that the sensitivity index $S_{5}$ rapidly decreases, the 
maximum sensitivity index along the $X$ direction is within 0.15 after the error levels are distributed, and the sensitivity indices of the remaining crucial geometric errors show a slight fluctuation and tend to be equivalent.

As shown in Figure 10, the sensitivity index of $h_{4}$ dramatically drops to 0.168 after the error levels are distributed, the maximum sensitivity index along the $Y$ direction is within 0.2 , and the sensitivity indices of the crucial geometric errors are nearly equivalent.

The sensitivity indices along the $Z$ direction and with respect to comprehensive error modeling are, respectively, shown in Figures 11 and 12. It is noted that all the sensitivity indices rapidly reduced to less than 0.2 after error distribution; it means that the error distribution method is effective to reduce the influence of the crucial geometric errors on the measurement result, which leads to the similar regularity obtained from Figures 9 and 10 .

From the sensitivity analysis result after error distribution, it is reasonably concluded that error distribution is effective to reduce the influence of the crucial geometric errors with large sensitivity index on measurement error. All the sensitivity indices rapidly decrease to near or below 0.2 , the crucial geometric errors having the most important influence along the $X, Y$, and $Z$ direction disappear, and the sensitivity indices of the crucial geometric errors tend to be nearly equivalent.

The sensitivity indices of the crucial geometric errors tend to be at the approximately equivalent level by the reasonable geometric error distribution and adjustment. The above results of sensitivity analysis are beneficial for the error distribution of the prototype of a 5-axis measuring machine, and the results provide significant meaning to the optimal design and accurate manufacturing of the 5-axis measuring machine, which is helpful to equalize the influence of the crucial geometric errors as possible.

4.3. Error Distribution and Adjustment Methods. Based on the detailed discussion of the forgoing sensitivity analysis result, the crucial geometric errors for the prototype of a 5axis measuring machine are three angular errors $\left(\theta_{x x}, \theta_{x y}\right.$, and $\theta_{x z}$ ) caused by the motion of $X$-axis, two angular errors $\left(\theta_{y x}\right.$ and $\left.\theta_{y y}\right)$ caused by the motion of $Y$-axis, three angular errors $\left(\theta_{z x}, \theta_{z y}\right.$, and $\left.\theta_{z z}\right)$ caused by the motion of $Z$-axis, and an angular error $\theta_{b y}$ caused by the motion of $B$-axis, as well as two squareness errors $\left(\beta_{z x}\right.$ and $\left.\beta_{y z}\right)$ of $Z$-axis to $X$-axis and $Y$-axis to $Z$-axis. It is summarized that the crucial geometric errors are composed of the angular errors caused by the translational axes, the angular error caused by the motion of $B$-axis, and the squareness errors.

Due to the characteristics of the crucial geometric errors, some practical measures can be taken to distribute and adjust the levels of the crucial geometric errors.

4.3.1. The Angular Errors of the Translational and Rotational Axes Can Be Reduced by Improving the Geometric Imperfections of the Manufacturing and Assembly of Machine Components. The translational axes ( $X$-axis, $Y$-axis, and $Z$ axis) and $B$-axis in the 5 -axis measuring machine are in the form of orifice-compensated hydrostatic bearing, as shown in Figure 13.

Improving the machining accuracy (dimensional accuracy and form accuracy) of the matching components is an effective approach to guarantee the matching clearance and oil film thickness. For a translational axis, the poor surface flatnesses for slideway carriages and slideway base are the main factors causing angular errors. For a rotational axis, the roundness errors and cylindricity errors of the shaft and shaft sleeve lead to the angular errors. Dimensional accuracy of the matching surfaces affects the oil film thickness for both a translational axis and a rotational axis, and the excessive oil film thickness causes the increasing angular errors. Therefore, these above form errors and dimensional errors need to be strictly monitored and corrected in the precision machining stage. Although the assembly accuracy is strongly dependent of the machining accuracy of the matching components, the assembly error induced in the assembly process can also cause the angular error, and the assembly inaccuracy should be fully considered to ensure the closure of the dimensional accuracy chain, which is beneficial to improve the angular errors of the translational and rotational axes.

Additionally, the increasing oil supply pressure is beneficial to reduce the angular errors because of the error averaging effect of the oil film. The oil film acts as a lubricant when a translational axis or a rotational axis is running, and the increasing oil supply pressure can directly improve the oil film stiffness and the error averaging effect, which is effective for decreasing the angular errors caused by the imperfections of the matching components [36].

4.3.2. The Squareness Errors $\left(\beta_{z x}\right.$ and $\left.\beta_{y z}\right)$ Can Be Improved by Adjusting the Corresponding Spatial Relationship among Axes and Software Correction Method. The squareness error of axis of motion with respect to each other is generally affected by the orientation of the reference line of these axes [26], and the squareness errors can be calibrated by the laser interferometer and double ball bar in the assembly process of machine components; therefore, adjusting the alignment and orientation of the reference line of these axes is helpful to enhance the squareness errors in the assembly process of the 5-axis measuring machine.

To overcome the accuracy limitation in the mechanical adjustment, the software correction method can be used to further improve the squareness errors. The software correction method uses UMAC (Universal Motion and Automation Controller) to redefine the coordinate system and correct the motor parameters in order to orthogonally compensate for the offset caused by the squareness errors.

The UMAC provides the function of creating a coordinate system, defining an axis system, and configuring the motor parameters. This function is employed in this study to perform orthogonal correction of the squareness errors $\beta_{z x}$ and $\beta_{y z}$.

The schematic of the squareness errors $\beta_{z x}$ and $\beta_{y z}$ is shown in Figure 14; the squareness error $\beta_{z x}$ causes the $x$ directional offset relative to the nominal position when $Z$ - 


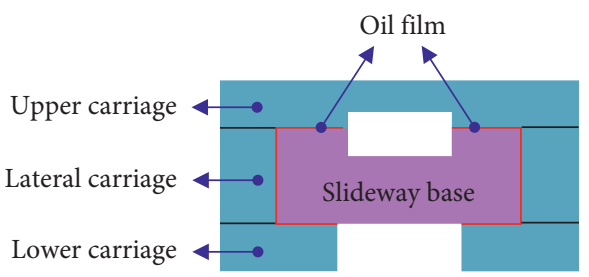

(a)

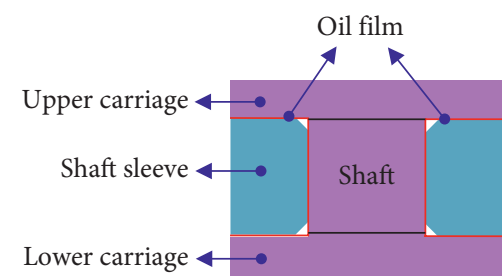

(b)

Figure 13: A schematic cross section of translational axes and $B$-axis: (a) the cross section of a translational axis; (b) the cross section of $B$ axis.

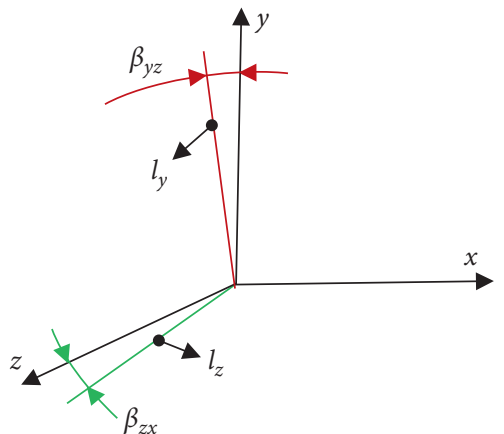

Figure 14: A schematic of squareness errors of $\beta_{z x}$ and $\beta_{y z} ; l_{y}$ represents the reference line of $Y$-axis, and $l_{z}$ represents the reference line of $Z$-axis.

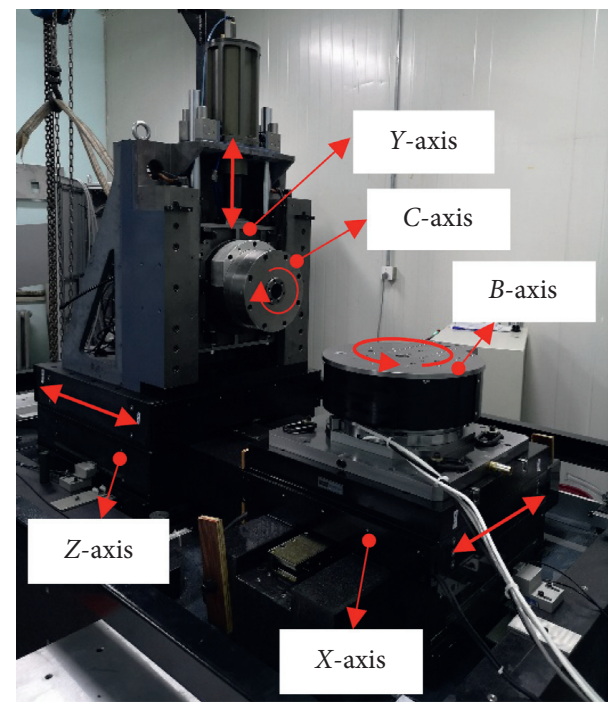

FIGURE 15: The experimental prototype of the 5-axis measuring machine.

axis is moving, and the squareness error $\beta_{y z}$ causes the $z$ directional offset relative to the nominal position when $Y$ axis is moving. The offset can be approximately represented by the reference line.

As the pulse signal is preferred by UMAC, the position of each axis should be first transformed into the corresponding number of pulse counts that can be recognized by UMAC. The linear encoder with the linear resolution of $5 \mathrm{~nm}$ is used as the position feedback unit in the 5-axis measuring machine, so the displacement of $1 \mathrm{~mm}$ corresponds to 200,000 pulse counts. The squareness errors $\beta_{z x}$ and $\beta_{y z}$ can be corrected by loading the following codes into UMAC:

Undefine All; \% Clear all axis-definitions in the coordinate system.

$\& 1 ; \%$ Use the symbol " $\&$ " to define a coordinate system and label it as No. 1 .

\#1->200000Y; \% Assign motor 1 to $Y$-axis, and convert the $Y$-axis position into the number of pulse counts.

$\# 2->200000 Z+200000 * Y * \tan \left(\beta_{y z}\right) ; \%$ Assign motor 2 to $Z$-axis, convert the $Z$-axis position into the number of pulse counts, correct the $z$-directional offset caused by the squareness errors $\beta_{y z}$.

\#3->200000X+200000*Z*tan $\left(\beta_{z x}\right) ; \%$ Assign motor 3 to $X$-axis, convert the $X$-axis position into the number of pulse counts, correct the $x$-directional offset caused by the squareness errors $\beta_{z x}$.

After the correction code has been stored in UMAC, the offset caused by the squareness errors can be compensated via a software correction method to improve the squareness errors $\beta_{z x}$ and $\beta_{y z}$.

The 5-axis measuring machine could be optimal designed and accurate manufactured for improving the efficiency and performance, and an experimental prototype is fabricated, as shown in Figure 15. The future work will mainly focus on the research of software development, measurement method, and data processing method.

\section{Conclusions}

The present work focuses on the error distribution of a 5-axis measuring machine based on sensitivity analysis of geometric errors. The objective of this paper is to equalize the influence of the crucial geometric errors on the measurement result by distributing reasonably the error levels of the crucial geometric errors. This ensures that the measurement accuracy of a 5-axis measuring machine is no longer strictly limited to some geometric errors with larger sensitivity index, which provides significant meaning to the optimal design and accurate manufacturing of the 5-axis measuring machine. The homogeneous coordinate transformation method was first employed to establish the measurement error modeling of the 5-axis measuring machine. The Sobol global sensitivity analysis method was then used to investigate the sensitivity indices of geometric errors and to 
further quantify the influence of geometric errors on the measurement result. Finally, the error levels of the crucial geometric errors were distributed and readjusted according to the sensitivity analysis result, and the sensitivity analysis result after error distribution demonstrates that the error distribution is effective to equalize the influence of the crucial geometric errors on the measurement result. Some practical approaches to distribute and improve the crucial geometric errors were given in detail.

A summary of the significant conclusions are presented as follows:

(1) The sensitivity analysis can investigate the influence of geometric errors on the measurement result in a 5axis measuring machine, which is beneficial for the accuracy design and manufacture of a 5-axis measuring machine. The sensitivity analysis result shows that most of the crucial geometric errors seriously affecting the measurement error are the angular errors caused by the translational axis and $B$-axis, as well as the squareness errors $\beta_{z x}$ and $\beta_{y z}$. The crucial geometric errors are supposed to pay more attention in the design and manufacturing stage.

(2) The error distribution method is proposed to equalize the influence of the crucial geometric errors on the measurement result, and the effectiveness of the proposed error distribution method is validated by the re-executed sensitivity analysis; some practical measures to distribute and adjust the levels of the crucial geometric errors are provided in detail.

(3) The error distribution method is effective to reduce the influence of the crucial geometric errors on the measurement result, and this finding is significant for the optimal design and accurate manufacturing of the 5-axis measuring machine.

\section{Data Availability}

The data used to support the findings of this study are included within the article.

\section{Conflicts of Interest}

The authors declare that they have no conflicts of interest.

\section{Acknowledgments}

The present work was supported by the NSAF (no. U1530106) and the National Natural Science Foundation of China (no. 51005061).

\section{Supplementary Materials}

(1) The supplementary material "the $X$-directional sensitivity indices of geometric errors before error distribution" provides the statistical results of the initial $X$-directional sensitivity indices of geometric errors at the 17 test points before error distribution, and the above sensitivity indices are represented by the column charts in Figure 5. (2) The supplementary material "the $Y$-directional sensitivity indices of geometric errors before error distribution" provides the statistical results of the initial $Y$-directional sensitivity indices of geometric errors at the 17 test points before error distribution, and the above sensitivity indices are represented by the column charts in Figure 6. (3) The supplementary material "the $Z$-directional sensitivity indices of geometric errors before error distribution" provides the statistical results of the initial $Z$-directional sensitivity indices of geometric errors at the 17 test points before error distribution, and the above sensitivity indices are represented by the column charts in Figure 7. (4) The supplementary material "sensitivity indices of geometric errors on volumetric measurement error before error distribution" provides the statistical results of the initial sensitivity indices of geometric errors on volumetric measurement error at the 17 test points before error distribution, and the above sensitivity indices are represented by the column charts in Figure 8. (5) The supplementary material "the $X$-directional sensitivity indices of geometric errors after error distribution" provides the statistical results of the $X$-directional sensitivity indices of geometric errors at the 17 test points after error distribution, and the above sensitivity indices are represented by the column charts in Figure 9. (6) The supplementary material "the $Y$-directional sensitivity indices of geometric errors after error distribution" provides the statistical results of the $Y$-directional sensitivity indices of geometric errors at the 17 test points after error distribution, and the above sensitivity indices are represented by the column charts in Figure 10. (7) The supplementary material "the $Z$-directional sensitivity indices of geometric errors after error distribution" provides the statistical results of the $Z$-directional sensitivity indices of geometric errors at the 17 test points after error distribution, and the above sensitivity indices are represented by the column charts in Figure 11. (8) The supplementary material "sensitivity indices of geometric errors on volumetric measurement error after error distribution" provides the statistical results of the sensitivity indices of geometric errors on volumetric measurement error at the 17 test points after error distribution, and the above sensitivity indices are represented by the column charts in Figure 12. (Supplementary Materials)

\section{References}

[1] C. Geng, Y. Wu, and J. Qiu, "Analysis of nonlinear error caused by motions of rotation axes for five-axis machine tools with orthogonal configuration," Mathematical Problems in Engineering, vol. 2018, Article ID 6123596, 16 pages, 2018.

[2] W. Tian, W. Gao, W. Chang, and Y. Nie, "Error modeling and sensitivity analysis of a five-axis machine tool," Mathematical Problems in Engineering, vol. 2014, Article ID 745250, 8 pages, 2014.

[3] S. Guo, D. Zhang, and Y. Xi, "Global quantitative sensitivity analysis and compensation of geometric errors of CNC machine tool," Mathematical Problems in Engineering, vol. 2016, Article ID 2834718, 12 pages, 2016.

[4] J. Wei and Y. Chen, "The geometric dynamic errors of CMMs in fast scanning-probing," Measurement, vol. 44, no. 3, pp. 511-517, 2011. 
[5] Y. Liu, M. Wan, W.-J. Xing, Q.-B. Xiao, and W.-H. Zhang, "Generalized actual inverse kinematic model for compensating geometric errors in five-axis machine tools," International Journal of Mechanical Sciences, vol. 145, pp. 299-317, 2018.

[6] H. Yao, Z. Li, X. Zhao, T. Sun, G. Dobrovolskyi, and G. Li, "Modeling of kinematics errors and alignment method of a swing arm ultra-precision diamond turning machine," The International Journal of Advanced Manufacturing Technology, vol. 87, no. 1-4, pp. 165-176, 2016.

[7] G. Chen, Y. Liang, Y. Sun, W. Chen, and B. Wang, "Volumetric error modeling and sensitivity analysis for designing a five-axis ultra-precision machine tool," The International Journal of Advanced Manufacturing Technology, vol. 68, no. 9-12, pp. 2525-2534, 2013.

[8] J. Li, F. Xie, and X.-J. Liu, "Geometric error modeling and sensitivity analysis of a five-axis machine tool," The International Journal of Advanced Manufacturing Technology, vol. 82, no. 9-12, pp. 2037-2051, 2016.

[9] Y. Liu, M. Wan, W.-J. Xing, and W.-H. Zhang, "Identification of position independent geometric errors of rotary axes for five-axis machine tools with structural restrictions," Robotics and Computer-Integrated Manufacturing, vol. 53, pp. 45-57, 2018.

[10] Y. Liu, M. Wan, Q.-B. Xiao, and W. H. Zhang, "Identification and compensation of geometric errors of rotary axes in fiveaxis machine tools through constructing equivalent rotary axis (ERA)," International Journal of Mechanical Sciences, vol. 152, pp. 211-227, 2019.

[11] E. B. Harper, J. C. Stella, and A. K. Fremier, "Global sensitivity analysis for complex ecological models: a case study of riparian cottonwood population dynamics," Ecological Applications, vol. 21, no. 4, pp. 1225-1240, 2011.

[12] A. Saltelli, P. Annoni, I. Azzini, F. Campolongo, M. Ratto, and S. Tarantola, "Variance based sensitivity analysis of model output. Design and estimator for the total sensitivity index," Computer Physics Communications, vol. 181, no. 2, pp. 259270, 2010.

[13] Q. Cheng, B. Sun, Z. Liu, J. Li, X. Dong, and P. Gu, "Key geometric error extraction of machine tool based on extended Fourier amplitude sensitivity test method," The International Journal of Advanced Manufacturing Technology, vol. 90, no. 9-12, pp. 3369-3385, 2016.

[14] M. Zhang, D. Djurdjanovic, and J. Ni, "Diagnosibility and sensitivity analysis for multi-station machining processes," International Journal of Machine Tools and Manufacture, vol. 47, no. 3-4, pp. 646-657, 2007.

[15] X. Zhang, Y. Zhang, and M. D. Pandey, "Global sensitivity analysis of a CNC machine tool: application of MDRM," The International Journal of Advanced Manufacturing Technology, vol. 81, no. 1-4, pp. 159-169, 2015.

[16] Q. Cheng, H. Zhao, G. Zhang, P. Gu, and L. Cai, "An analytical approach for crucial geometric errors identification of multi-axis machine tool based on global sensitivity analysis," The International Journal of Advanced Manufacturing Technology, vol. 75, no. 1-4, pp. 107-121, 2014.

[17] ISO 841, Industrial Automation Systems and IntegrationNumerical Control of Machines-Coordinate System and Motion Nomenclature, International Organization for Standardization, Geneva, Switzerland, 2001.

[18] X. Liu, X. Zhang, F. Fang, Z. Zeng, H. Gao, and X. Hu, "Influence of machining errors on form errors of microlens arrays in ultra-precision turning," International Journal of Machine Tools and Manufacture, vol. 96, pp. 80-93, 2015.
[19] X. Liu, X. Zhang, F. Fang, and S. Liu, "Identification and compensation of main machining errors on surface form accuracy in ultra-precision diamond turning," International Journal of Machine Tools and Manufacture, vol. 105, pp. 4557, 2016.

[20] S.-H. Yang, K.-H. Kim, Y. K. Park, and S.-G. Lee, "Error analysis and compensation for the volumetric errors of a vertical machining centre using a hemispherical helix ball bar test," The International Journal of Advanced Manufacturing Technology, vol. 23, no. 7-8, pp. 495-500, 2004.

[21] H. Schwenke, W. Knapp, H. Haitjema, A. Weckenmann, R. Schmitt, and F. Delbressine, "Geometric error measurement and compensation of machines-An update," CIRP Annals, vol. 57, no. 2, pp. 660-675, 2008.

[22] ISO 230-1, Test Code for Machine Tools-Part 1: Geometric Accuracy of Machines Operating under No-Load or QuasiStatic Conditions, International Organization for Standardization, Geneva, Switzerland, 2012.

[23] ISO 230-2, Test Code for Machine Tools-Part 2: Determination of Accuracy and Repeatability of Positioning Numerically Controlled Axes, International Organization for Standardization, Geneva, Switzerland, 2006.

[24] ISO 230-7, Test Code for Machine Tools-Part 7: Geometric Accuracy of Axes of Rotation, International Organization for Standardization, Geneva, Switzerland, 2015.

[25] ISO/IEC Guide 98-3, Uncertainty of Measurement-Part 3: Guide to the Expression of Uncertainty in Measurement, International Organization for Standardization, Geneva, Switzerland, 2008.

[26] ISO 16907, Machine Tools-Numerical Compensation of Geometric Errors, International Organization for Standardization, Geneva, Switzerland, 2015.

[27] Z. Li, R. Sato, K. Shirase, Y. Ihara, and D. S. Milutinovic, "Sensitivity analysis of relationship between error motions and machined shape errors in five-axis machining center- peripheral milling using square-end mill as test case," Precision Engineering, vol. 60, pp. 28-41, 2019.

[28] A. Saltelli, M. Ratto, F. Campolongo, J. Cariboni, D. Gatelli, and M. Saisana, Global Sensitivity Analysis-The Primer, Wiley, Chichester, England, 2008.

[29] I. M. Sobol, "Global sensitivity indices for nonlinear mathematical models and their Monte Carlo estimates," Mathematics and Computers in Simulation, vol. 55, no. 1-3, pp. 271-280, 2001.

[30] F. Pianosi and T. Wagener, "A simple and efficient method for global sensitivity analysis based on cumulative distribution functions," Environmental Modelling \& Software, vol. 67, pp. 1-11, 2015.

[31] A. Saltelli, S. Tarantola, and K. P.-S. Chan, "A quantitative model-independent method for global sensitivity analysis of model output," Technometrics, vol. 41, no. 1, pp. 39-56, 1999.

[32] F. Ferretti, A. Saltelli, and S. Tarantola, "Trends in sensitivity analysis practice in the last decade," Science of the Total Environment, vol. 568, pp. 666-670, 2016.

[33] I. M. Sobol, "Quasi-Monte Carlo methods," Progress in $\mathrm{Nu}$ clear Energy, vol. 24, no. 1-3, pp. 55-61, 1990.

[34] A. Saltelli, S. Tarantola, and K. Chad, "Presenting results from model based studies to decision-makers: can sensitivity analysis Be a defogging agent?," Risk Analysis, vol. 18, no. 6, pp. 799-803, 1998.

[35] X. Zou, X. Zhao, G. Li, Z. Li, and T. Sun, "Sensitivity analysis using a variance-based method for a three-axis diamond turning machine," The International Journal of Advanced 
Manufacturing Technology, vol. 92, no. 9-12, pp. 4429-4443, 2017.

[36] E. Qi, Z. Fang, T. Sun, J. Chen, C. Liu, and J. Wang, "A method for predicting hydrostatic guide error averaging effects based on three-dimensional profile error," Tribology International, vol. 95, pp. 279-289, 2016. 